\title{
Technological Advances in Applied Intelligence
}

\author{
Malek Mouhoub, Samira Sadaoui, \\ Otmane Ait Mohamed, Moonis Ali
}

- The 31st International Conference on Industrial, Engineering and Other Applications of Applied Intelligent Systems (IEA/AIE 2018) was held at Concordia University in Montreal, Canada, June 25-28, 2018. IEA/AIE 2018 continued the tradition of emphasizing applications of applied intelligent systems to solve real-life problems in all areas, including engineering, science, industry, automation and robotics, business and finance, medicine and biomedicine, bioinformatics, cyberspace, and human-machine interactions.
$\mathrm{T}$ he annual IEA/AIE conference began in 1988, in Tullahoma, Tennessee, and has since been held in many countries across the globe, including Germany, Scotland, Australia, Canada, Spain, Egypt, Hungary, England, Italy, France, Japan, Poland, China, Taiwan, Netherlands, and South Korea. As in past years, the conference was sponsored by the International Society of Applied Intelligence (ISAI) and held in cooperation with AAAI, SIGAI/ACM, and several other international organizations. The conference provides a medium for presenting, publishing, and communicating the scientific research and technological achievements accomplished by the international community. The 2018 conference was cochaired by general cochairs Otmane Ait Mohamed and Moonis Ali and program cochairs Malek Mouhoub and Samira Sadaoui.

IEA/AIE 2018 received 146 submissions from Canada and the following 44 countries: Algeria, Argentina, Australia, Austria, Belgium, Brazil, Chile, China, Cuba, the Czech Republic, Egypt, France, Germany, Greece, Hong Kong, India, Iran, Iraq, Italy, Japan, Jordan, Luxembourg, Malaysia, Morocco, The Netherlands, New Zealand, Nigeria, Norway, Oman, Pakistan, Poland, Portugal, Saudi Arabia, Singapore, Spain, Sweden, Switzerland, Taiwan, Tanzania, Tunisia, Turkey, the UAE, the UK, and the USA. Each submission was carefully reviewed by three members of the program committee.

The committee selected 64 papers for oral presentation at 
the conference and later publication in the proceedings, which were published by Springer in the Lecture Notes in Artificial Intelligence (LNCS/LNAI) series. The papers covered a wide range of artificial intelligence topics with a focus on machine learning, including knowledge representation, natural language processing, robotics, planning and scheduling, constraint programming, and evolutionary computation. In addition, 22 papers were selected (from the papers submitted directly to the special session chairs) for presentation at the following four special tracks and for inclusion in the proceedings: the Artificial Intelligence, Law, and Justice track; the Data Science, Privacy, and Security track; the Intelligent Systems Approaches in Information Extraction track; and the Internet of Things and Ubiquitous Computing and Big Data track.

In addition to the scheduled sessions and special tracks, three keynote talks, a tutorial, and social activities were organized. Among the activities were the conference reception, which took place at the Grevin Montreal Museum, and the Gala Dinner at the museum.

\section{Keynote Presentations}

The conference program was enriched by three keynote presentations. In "Technologies and Prospects of Autonomous and Connected Vehicles," Behrouz Far (University of Calgary) discussed the issues and challenges of autonomous and connected vehicles and explored the prospects of intelligent transportation systems. As part of the discussion, Far covered the key enabling technologies for practical implementation of autonomous ground vehicles (AGVs).

In his talk, "On the Path towards Intelligent Logging Systems," Wahab Hamou-Lhadj (Concordia University) canvassed state of the art research in system logging and monitoring and discussed the work he's currently involved in, designing intelligent logging and monitoring capabilities based on big data and machine learning. He also introduced the concept of designing for monitorability, which aims to bring logging to the early stages of the software development lifecycle.

In "Question-Answering Systems: Challenges and Perspectives," Guy Lapalme (University of Montreal) put the approaches of recent systems into perspective with a survey of question-answering techniques over the past 20 years. Lapalme then discussed the main challenges in building such systems and the need for more data.

\section{Tutorial}

The IEA/AIE 2018 conference also included a tutorial, "Accelerating Data Analytics Using Hadoop, Spark, and TensorFlow," presented by Behrouz Far
(University of Calgary) and Emad Amin Mohammed (Lakehead University). The tutorial walks users in detail through the steps required to install and configure an environment for big data and deep learning. The presenters also showed attendees how to use TensorFlow and Spark together to train and apply deep learning models to build a data science project.

\section{Best Paper Awards}

Three papers were selected for the Best Paper awards. The recipients of these awards received a monetary prize and an award certificate from Springer in addition to a certificate from ISAI. The selection committee included Bing Zhou (chair) from Sam Houston State University, USA, Pawan Lingras from Saint Mary's University, Canada, and Mohamed El Bachir Menai from King Saud University, Saudi Arabia.

The first-place winners and recipient of a $€ 500$ prize were Nuha Zamzami and Nizar Bouguila for their paper "Text Modeling Using Multinomial Scaled Dirichlet Distributions."

The second-place winners (and recipient of a $€ 300$ prize) were Amir Hosseinmemar, John Anderson, Jacky Baltes, Meng Cheng Lau, and Chi Fung Lun for "Closed-Loop Push Recovery for an Inexpensive Humanoid Robot."

In third place (and recipient of a $€ 200$ prize) were Kyle J. Morris, Vladyslav Samonin, John Anderson, Meng Cheng Lau, and Jacky Baltes for "Robot Magic: A Robust Interactive Humanoid Entertainment Robot."

\section{The 2019 Conference}

The 32nd International Conference on Industrial, Engineering and Other Applications of Applied Intelligent Systems will continue the tradition of emphasizing applications of applied intelligent systems to solve real-life problems in all domains, such as engineering, science, industry, automation and robotics, business and finance, medicine and biomedicine, bioinformatics, cyberspace, and human-machine interactions, and other applicable fields. In addition, IEA/AIE 2019 will have a special focus on automated driving and autonomous systems. Contributions dealing with such systems or their verification and validation are especially welcome.

Moonis Ali is a professor of computer science at Texas State University.

Otmane Ait Mohamed is a professor of electrical and computer engineering at Concordia University.

Malek Mouhoub is a professor in the Department of Computer Science at the University of Regina.

Samira Sadaoui is a professor of computer science at the University of Regina. 